\title{
Characteristics of a Semiconductor Laser with External Feedback
}

\author{
Hiroshi Kakiuchida and Junji Ohtsubo
}

\begin{abstract}
The theoretical analysis of the compound cavity of a semiconductor laser with external optical feedback is conducted. For large optical feedback, the output power from the laser and its oscillation frequency differ from those for small optical feedback. From the rate equations of the compound cavity, the conditions of the laser oscillation are derived in the presence of large optical feedback. The dependencies of the output power and the laser oscillation frequency on the external-cavity length are investigated. Some new results involving laser oscillation depending on the external-cavity length are presented. The experimental results are compared with theoretical predictions. The dependence of the laser oscillation on the external-cavity length is qualitatively explained in the present model.
\end{abstract}

\section{INTRODUCTION}

I N MANY applications of a semiconductor laser, external optical feedback to a laser cavity plays an important role in the performance of the system. In some cases, it induces noise and greatly degrades the capability of information transmission, such as in optical-fiber communications, optical-data storage, and various optical measurements [1]. It is important to know the characteristics of a semiconductor laser with external optical feedback in such systems. On the other hand, it can be applied for practical use in optical measurements, for example, optical-mixing frequency-shifting LDVs [2], [3] and spectral narrowing by a mode selection [4]. Therefore, the characteristics of semiconductor lasers with external optical feedback have attracted much attention from many researchers since their invention [5]-[9].

It has been already pointed out that external optical feedback affects the oscillation characteristics and the oscillation condition is dependent upon the distance between a semiconductor laser and an external reflector [5], [6]. The laser-oscillation conditions are changed by shortrange variations of the external-cavity length of the order of wavelength $\lambda$ of light. However, there also exists a change in the oscillation conditions for long-range variations of the order of the external-cavity length (the effective laser cavity length equal to $\sim 1 \mathrm{~mm}$ for an ordinary

Manuscript received June 21, 1993; revised June 21, 1993. This work was supported by the Mechanical Industry Development and Assistance Foundation (MIDAF).

H. Kakiuchida was with the Department of Engineering, Shizuoka University, Hamamatsu 432, Japan. He is now with the Dainippon Screen Co. Ltd., Kamigyoku, Kyoto 602, Japan.

J. Ohtsubo is with the Faculty of Engineering, Shizuoka University, Hamamatsu 432, Japan.

IEEE Log Number 9402511. semiconductor laser). The characteristics of laser oscillation both for short- and long-range variations have been reported in several papers [5]-[8]. However, the oscillation characteristics, especially for the long-range variations of the cavity length, are still not clarified. Furthermore, most of the papers concerned with external optical feedback only treat a small back reflection of light in the external cavity (i.e., a single reflection) because of its simplicity. Osmundsen and Gade [5] and others [3] discussed multiple reflections in the external cavity and obtained some useful results. But few papers have presented experimental results for multiple reflections and conducted a comparison of theory with experiments.

In this paper, we discuss the characteristics of a semiconductor laser with an external cavity and the dependencies of the laser oscillation on the amount of external optical feedback and the variations of the external-cavity length. Here, we discuss the fact that the distance of the external mirror from the laser cavity is much smaller than the coherence length and the external-reflector system behaves as a laser with a compound cavity. The theoretical background is presented by using the rate equations in which the effects of multiple reflections of light by the external cavity are taken into consideration. We introduce the compound-cavity model for such a system. It is shown that the effects of the compound cavity result in an additional gain factor and a phase change in the theoretical derivations. Based on these discussions, the output power and oscillation frequency of a laser with external feedback are obtained as a function of the injection current. Further, we consider the multimode oscillation of a laser with external feedback and try to explain the dependence of the output-power variation on the external-cavity length that is observed in the experiments. Some new experimental results are presented for ratherstrong optical feedback. We also try to explain the obtained results from the point of view of the agreement and disagreement between the internal and external modes. We ignore mode competition in multimode oscillations in the discussion, allowing that our discussion may not be perfect. Despite these drawbacks, the theoretical results agree well with the experimental findings.

\section{THEORY}

\section{A. Rate Equations}

Fig. 1 shows the schematic illustration of a cavity model for the semiconductor laser with external optical feedback 


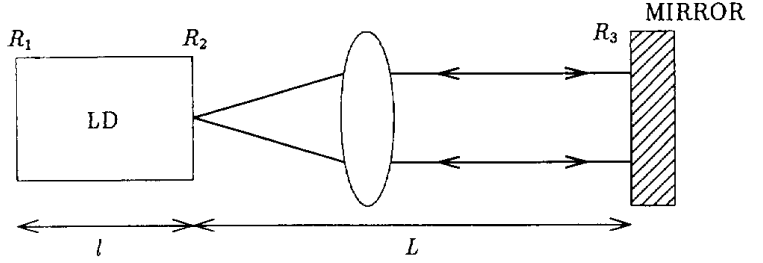

Fig. 1. Cavity model for a semiconductor laser with external optical feedback.

that we treat here. The output power from a laser cavity (length $l$ and refractive index $\eta$ ) is reflected by an external mirror (intensity reflectance $R_{3}$ ), which is located at a distance $L$ from the front facet of the laser cavity. The reflected light is directed by feedback into the laser cavity. The internal-intensity reflectances of the front and rear facets are $R_{2}$ and $R_{1}$, respectively. The rate equations for such a semiconductor laser with external optical feedback have already been derived and extensively studied by Lang and Kobayashi [5]. However, they only treated the case of small optical feedback and neglected multiple reflections in the external cavity. The case we treat here is for large optical feedback so that we must take account of the higher order multiple reflection terms in the rate equations. In this section, we briefly discuss the rate equations for multiple optical feedbacks and derive the conditions for laser oscillation.

The rate equation for the complex field $E(t) \exp (i \Omega t)$ at time $t$ is written by

$$
\begin{aligned}
& \frac{d}{d t} E(t) \exp (i \Omega t) \\
& \quad=\left[i \omega_{N}+\frac{1}{2}\left(G-\Gamma_{w}\right)+\beta(t)\right] E(t) \exp (i \Omega t)
\end{aligned}
$$

where $\Omega$ and $\omega_{N}$ are the angular frequencies of the laser oscillation and the $N$ th laser cavity mode, respectively, $G$ is the gain coefficient, and $\Gamma_{w}$ is the loss in the laser cavity. The other counterpart of the rate equations is for the carrier density $n(t)$ and is given by

$$
\frac{d}{d t} n(t)=J-\gamma n(t)-G|E(t)|^{2}
$$

where $J$ is the density of the injection carriers and $\gamma$ is the inverse of the spontaneous lifetime of the excited carriers. The time-dependent function $\beta(t)$, which is related to the optical feedback including the reflection losses of the laser facets and the external mirror, is introduced in (1). It is a complex function and written by

$$
\beta(t)=-\frac{1}{2} \beta_{r}(t)+i \beta_{i}(t) .
$$

In (3), the coefficient of $-\frac{1}{2}$ in the real part is chosen for simplicity later. It is proved in the following discussion that the real part of $\beta(t)$ represents the additional gain due to optical feedback, while the imaginary part denotes the phase shift of the laser frequency. The function $\beta(t)$ is time dependent, but it only becomes a function of the round-trip time $\tau$ of light in the external cavity under steady-state conditions.

\section{B. Conditions of Laser Oscillation with an External Cavity}

We now consider a laser cavity with external optical feedback as a compound cavity, as shown in Fig. 2. The reflectances of the front facet and the external mirror and the effect of the phase delay of light due to the external cavity are replaced by the effective reflectance $r_{\text {eff }}$ at the front facet. Then, the complex gain $\exp \left(\beta \tau_{D}\right)$ should coincide with the total loss of the compound-mirror reflections $r_{1} r_{\text {eff }}$ at the laser threshold, i.e.,

$$
\exp \left(\beta \tau_{D}\right)=r_{1} r_{\mathrm{eff}}
$$

where $r_{1}$ (the corresponding intensity reflectance is $R_{1}$ ) and $r_{\text {eff }}$ are defined as amplitude reflectances. We consider a steady-state condition so that the time $t$ of the function $\beta(t)$ is replaced by the round-trip time $\tau_{D}$ in the laser cavity. The round-trip time in the laser cavity is given by

$$
\tau_{D}=\frac{2 \eta l}{c}
$$

where $c$ is the speed of light in vacuum. The effective complex reflectance $r_{\text {eff }}$ is derived from the consideration of the multiple reflections of light in the external cavity. The effective reflectivity has already been calculated and given by [6]

$$
r_{\text {eff }}=\frac{r_{2}+r_{3} \exp (-i \Omega \tau)}{1+r_{2} r_{3} \exp (-i \Omega \tau)}
$$

where $r_{2}$ and $r_{3}$ (the corresponding intensity reflectances are $R_{2}$ and $R_{3}$ ) are the amplitude reflectances of the front facet of the laser cavity and the external mirror, respectively, and $\tau$ is the round-trip time of light in the external cavity and given by

$$
\tau=\frac{2 L}{c}
$$

Substituting (6) into (4), one obtains the following relation:

$$
\exp \left(\beta \tau_{D}\right)=\exp \left[-\ln \left(\frac{1}{r_{1} r_{2}}\right)\right] \frac{1+r_{3} / r_{2} \exp (-i \Omega \tau)}{1+r_{2} r_{3} \exp (-i \Omega \tau)}
$$

Then, the real and imaginary parts of the complex gain factor $\beta$ are written by

$$
\begin{aligned}
& \beta_{r}=\frac{2}{\tau_{D}} \ln \left(\frac{1}{r_{1} r_{2}}\right)+\frac{2}{\tau_{D}} g\left(r_{3}, \tau, \Omega\right) \\
& \beta_{i}=\frac{1}{\tau_{D}} \phi\left(r_{3}, \tau, \Omega\right)
\end{aligned}
$$




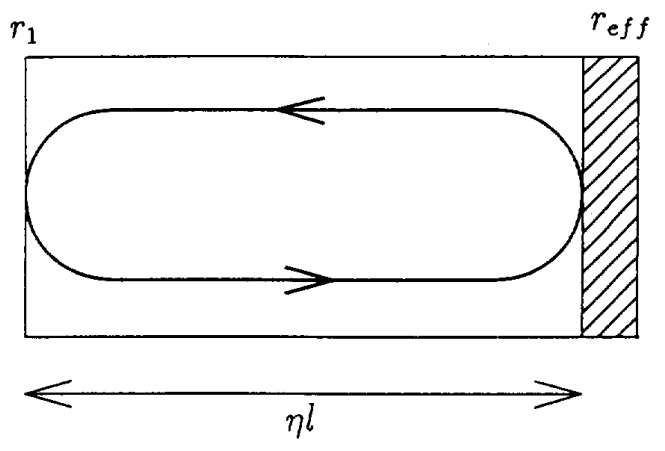

Fig. 2. Schematic illustration of the compound cavity.

respectively, where $g$ and $\phi$ are

$g\left(r_{3}, \tau, \Omega\right)=\frac{1}{2} \ln \left[\frac{1+2 r_{2} r_{3} \cos (\Omega \tau)+\left(r_{2} r_{3}\right)^{2}}{1+2 r_{3} / r_{2} \cos (\Omega \tau)+\left(r_{3} / r_{2}\right)^{2}}\right]$

(10a)

$\phi\left(r_{3}, \tau, \Omega\right)=\arctan \left[\frac{\left(r_{2} r_{3}-r_{3} / r_{2}\right) \sin (\Omega \tau)}{1+r_{3}^{2}+\left(r_{2} r_{3}+r_{3} / r_{2}\right) \cos (\Omega \tau)}\right]$.

We now consider the condition of laser oscillation in the compound cavity. At steady-state oscillation, we can determine the condition of the laser oscillation at the threshold from the rate equations and the relation in (3) as

$$
\frac{1}{2}\left(G_{\mathrm{th}}-\Gamma_{w}-\beta_{r}\right)+i\left(\omega_{N}-\Omega+\beta_{i}\right)=0
$$

where $G_{\text {th }}$ is the threshold gain. It is recognized from above equation that $\beta_{r}$ and $\beta_{i}$ are the additional threshold gain and the phase shift due to the presence of the external cavity, respectively. Then, the threshold gain and the oscillation frequency are written by

$$
\begin{aligned}
G_{\mathrm{th}} & =\Gamma_{w}+\beta_{r}=\Gamma_{0}+\frac{2}{\tau_{D}} g\left(r_{3}, \tau, \Omega\right) \\
\Omega & =\omega_{N}+\beta_{i}=\omega_{N}+\frac{1}{\tau_{D}} \phi\left(r_{3}, \tau, \Omega\right)
\end{aligned}
$$

where $\Gamma_{0}$ is the loss of the cavity without external optical feedback and given by

$$
\Gamma_{0}=\Gamma_{w}+\frac{2}{\tau_{D}} \ln \left(\frac{1}{r_{1} r_{2}}\right)
$$

From the above equations, the conditions for the threshold gain and laser oscillation with external optical feedback are determined and the characteristics of the compound cavity are investigated.

\section{Output Power and Oscillation Frequency}

The laser output power is linearly proportional to the injection current and increases with an increase of the injection current when there is no external feedback.
However, this linearity is lost in the compound cavity. Under steady-state conditions, the laser-output power $P$ with external optical feedback is calculated by solving the carrier-density rate equation (2) and is given as a function of the injection current $I$ by

$$
P=\mu \frac{\Omega}{G_{\mathrm{th}}}\left(I-I_{\mathrm{th}}\right)
$$

where $I_{\text {th }}$ is the injection current at laser threshold. The coefficient $\mu$ is determined by the device parameters in the laser cavity and written by

$$
\mu=\frac{\Gamma \epsilon h}{4 \pi \tau_{M} q}
$$

where $\Gamma$ is the confinement factor, $\epsilon$ is the injection efficiency, $h$ is Planck's constant, $\tau_{M}$ is the lifetime of light due to the reflection losses, and $q$ is the electric charge. Without external optical feedback, the oscillation frequency $\Omega$ and the threshold gain $G_{\text {th }}$ are constant, so that the laser output power is linearly proportional to the injection current above threshold. But with external optical feedback, the oscillation frequency and the threshold gain vary depending on the external-feedback power and the external-cavity length.

Next, we consider the oscillation frequency of the laser. The laser oscillation depends on the refractive index in the active layer. The refractive-index change is induced by changes of the carrier density and the temperature via an increase or decrease of the injection current. At steady state, the refractive index is linearized as a function of the injection current for small-current variations. Then, the refractive index is written by [5]

$$
\eta(I)=\eta_{0}+\eta_{r}\left(I-I_{0}\right)
$$

where $\eta_{0}$ is the refractive index at a reference injection current $I_{0}$ and $\eta_{r}$ is the proportional coefficient. The angular frequency $\omega_{N}$ of the laser-cavity mode depends on the refractive index and is given by

$$
\omega_{N}=\frac{N \pi c}{\eta(I) l} .
$$

For a small-refractive-index change (actually, the change of the refractive index is as small as of the order of $10^{-5}$ ), the angular frequency is approximated by

$$
\omega_{N} \approx \omega_{N 0}-2 \pi I_{r}\left(I-I_{0}\right)
$$

where $\omega_{N 0}$ is the standard-resonant-angular frequency

$$
\omega_{N 0}=\frac{N \pi c}{\eta_{0} l}
$$

and $I_{r}$ is the frequency-modulation efficiency

$$
I_{r}=\frac{N \eta_{r} c}{2 \eta_{0}^{2} l}
$$


The oscillation frequency becomes not only a function of the parameters of the external-cavity length, but also of the injection current. Substituting (18) into (12b), the phase condition is calculated and given by

$$
\Omega=\omega_{N 0}+\frac{1}{\tau_{D}} \phi\left(r_{3}, \tau, \Omega\right)-2 \pi I_{r}\left(I-I_{0}\right) .
$$

Solving (21), the relation between the oscillation frequency and the injection current can be determined. The threshold gain is obtained from the gain condition of (12a). Then, the laser output power is calculated by substituting the obtained angular frequency $\Omega$ and the threshold gain $G_{\text {th }}$ into (14). Fig. 3 shows the calculated threshold gain and the oscillation frequency with and without external feedback as a function of the resonant frequency. In the figure, the axis of the threshold gain is normalized by that with no external feedback as $G_{\mathrm{th}} / G_{\mathrm{th} 0}$ and the axes of the oscillation and resonant frequencies are also normalized as $\Omega \tau_{D} / 2 \pi$ and $\omega_{N} \tau_{D} / 2 \pi$, respectively. It is easily recognized from (18) that the resonant frequency is proportional to the injection current. Therefore, the vertical axis in the figure equivalently represents the injection current. Fig. 3 (a) shows the case for no external optical feedback. The oscillation frequency is linearly proportional to the injection current in this case. On the other hand, the linear relation is lost in Fig. 3 (b) and (c) due to the presence of external feedback, and the threshold gain varies periodically with the resonant frequency change. At large optical feedback, both the threshold gain and the oscillation frequency show bistable states, as shown in Fig. 3 (c).

\section{Dependence of Output Power on External-Cavity Length}

For a single-mode laser, laser oscillation undergoes a periodic change depending on variations of the externalcavity length equal to $\lambda / 2$, which is equivalent to the phase change of $2 \pi$ in the external cavity. In our experiments, except for this short-range periodic structure, we observed output-power undulation, which has a fundamental period of the effective internal-laser-cavity length $\eta l$. Further, with an increase of external feedback power, several subdips within the fundamental period are observed. It is difficult to explain this effect for laser oscillation under single-mode operation. To explain this effect, we consider two- or many-mode oscillations for the semiconductor laser. In the following discussion, we assume that the modes are independent of each other and neglect mode competition among each oscillation mode. Such a treatment is not always true under actual conditions of laser oscillation [10]. However, the qualitative discussion is possible and the experimentally observed phenomena can be well explained by such a model.

For independent two-oscillation modes, the threshold gain for each mode is written by

$$
\begin{gathered}
G_{N \text { th }}=\Gamma_{0}+\frac{2}{\tau_{D}} g\left(r_{3}, \tau, \Omega_{N}\right) \quad \text { (for } N \text { th mode) } \\
G_{N^{\prime} \text { th }}=\Gamma_{0}+\frac{2}{\tau_{D}} g\left(r_{3}, \tau, \Omega_{N^{\prime}}\right) \quad \text { (for } N^{\prime} \text { th mode) }
\end{gathered}
$$

where $\Omega_{N}$ and $\Omega_{N^{\prime}}$ are the oscillation angular frequencies of the $N$ th and $N^{\prime}$ th modes, respectively. As shown in (14), the laser output power $P$ is inversely proportional to the threshold gain, so that the maximum of the power occurs at the minimum of the threshold gain. The conditions of the minimum thresholds for the two modes are obtained from (10a) as

$$
\begin{aligned}
& \Omega_{N} \tau=2 M \pi \\
& \Omega_{N^{\prime}} \tau=2 M^{\prime} \pi
\end{aligned}
$$

where $M$ and $M^{\prime}$ are integers. Under the condition satisfied in (23), one obtains the minimum-threshold gains

$$
G_{N \text { th }}=G_{N^{\prime} \text { th }}=\frac{2}{\tau_{D}} \ln \left(\frac{1+r_{2} r_{3}}{1+r_{3} / r_{2}}\right) .
$$

We now assume that the öscillation angular frequency of each mode is approximately equal to the resonant angular frequency, that is,

$$
\begin{gathered}
\Omega_{N} \approx \frac{N \pi c}{\eta l} \\
\Omega_{N^{\prime}} \approx \frac{N^{\prime} \pi c}{\eta l} .
\end{gathered}
$$

These conditions together with (23) indicate that the maximum of each mode power occurs when the externalcavity length $L$ is equal to integer multiples of the effective-cavity length $\eta l$.

From the above discussion, the maximum power of the laser oscillation is realized when the following condition is satisfied:

$$
\frac{L}{\eta l}=\frac{m}{n}
$$

where $m=M^{\prime}-M$ and $n=N^{\prime}-N$. When $N^{\prime}=N+$ 1 , which is possible in this case, this relation reduces to $L / \eta l=m$. This means that the maximum output power is attained at the external-cavity length equal to integer multiples of the effective-laser cavity length $\eta l$. The total output power at the maximum condition is calculated from a linear summation of each mode power as

$$
P \approx \mu \omega_{N 0}\left(\frac{1}{G_{N \text { th }}}+\frac{1}{G_{N^{\prime} \text { th }}}\right)\left(I-I_{\mathrm{th}}\right) .
$$

Fig. 4 shows the calculated result of the output power as a function of the external-cavity length $L$ for two-mode oscillation at $n=1$. The curves are plotted for the different values of the feedback-intensity reflectance. The maximum power occurs at integer multiples of the effective-laser-cavity length and the dips of the output power are seen at increments equal to half of the effective-cavity length. It is noted the large dips in the output power are realized at rather-moderate optical-feedback intensities. 

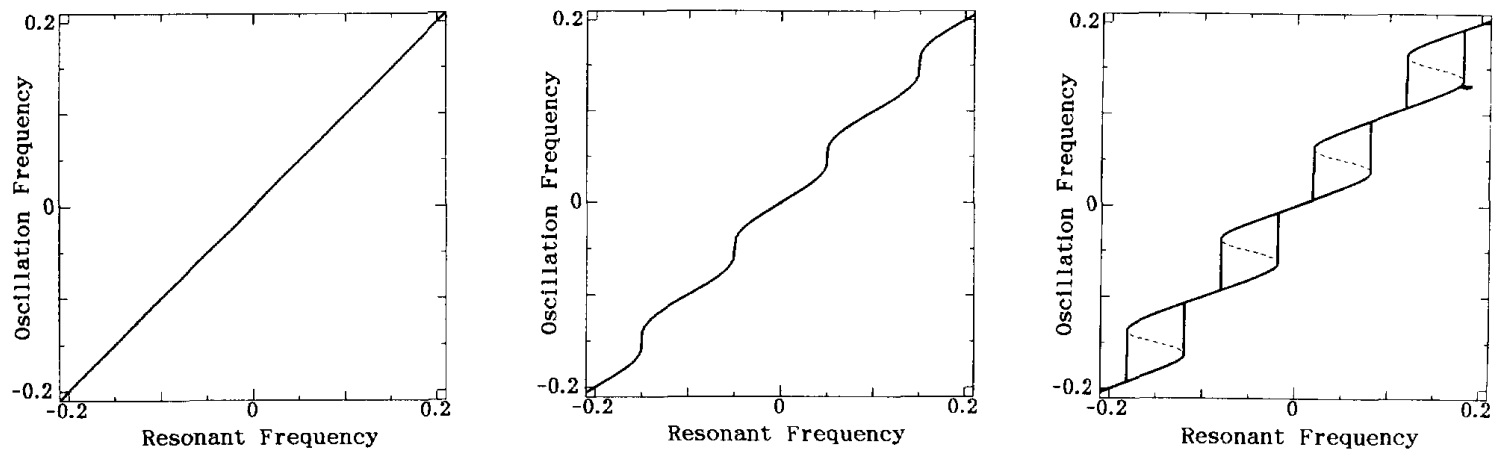

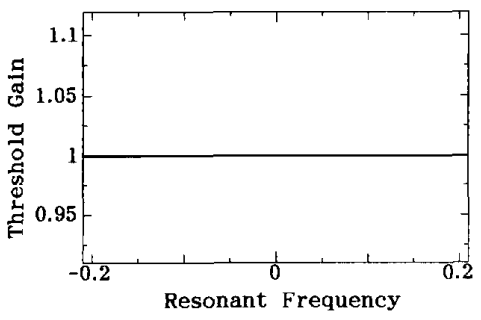

(a)

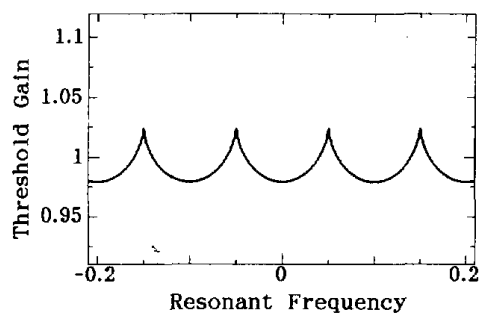

(b)

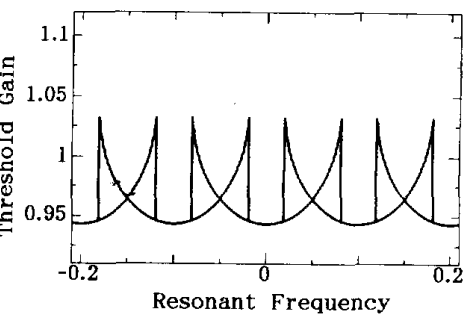

(c)

Fig. 3. Oscillation frequency and threshold gain as a function of the resonant frequency for different intensity reflectances. (a) $R_{3}=0$, (b) $R_{3}=0.005$, and (c) $R_{3}=0.05$.

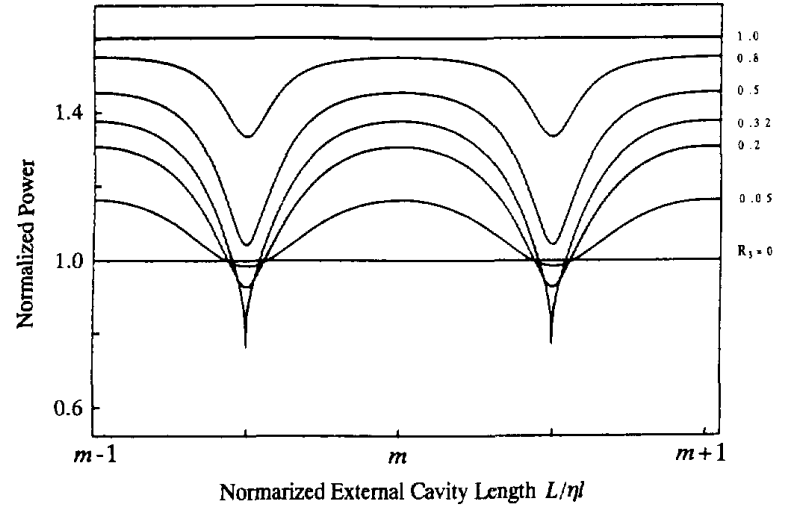

Fig. 4. Output power as a function of the external-cavity length.

In the experiments, several subdips are observed between the maximum powers when the intensity from the external reflector is enhanced. The effect is explained by the multimode oscillation between the distant modes or the multimode oscillation in more than two modes. It is obvious that the total output power at a multimode oscillation of more than two modes is calculated from the summation over all the respective intensities.

\section{EXPERIMENTS AND DISCUSSIONS}

The experimental setup for studying the characteristics of a semiconductor laser with external optical feedback is shown in Fig. 5. The semiconductor laser used is a singlemode CSP laser diode (Hitachi HL7801E), which oscillated at a wavelength of $0.780 \mu \mathrm{m}$ and a maximum power of $5 \mathrm{~mW}$. The threshold current of the laser was $50 \mathrm{~mA}$. The temperature of the laser was stabilized by an automatic-temperature-control (ATC) circuit. The injection current to the laser can be modulated by an alternatingcurrent drive circuit. The emitted light from the laser diode (LD) was collimated by a lens (L) and split by a beamsplitter (BS). One of the beams was reflected by an external mirror (M) through a variable neutral density filter ( $v$-ND) and fed back to the laser cavity. The mirror was mounted on a motor-driven moving stage controlled by a microcomputer. The step of the control was $4 \mu \mathrm{m}$. The other beam was fed to the frequency-measuring system. The oscillation frequency from the laser was investigated by the system. The output power emitted from the rear facet of the laser cavity was monitored by a photodiode (PD) installed within the laser-diode package. We adopted two types of frequency measuring systems in the experiments. One was a Fabry-Perot interferometer and the other was an unbalanced Twyman-Green interferometer.

It is very difficult to determine the exact amount of external-feedback intensity to the laser cavity. In the actual experiments, we simply employed the value of the fraction of the feedback intensity at the exit face of the lens ( $L$ ) for the intensity emitted from the lens as an external reflectance. The lens was the objective lens of a microscope and its magnification and numerical aperture were $\times 1.0$ and $\times 0.25$, respectively. The power emitted from the laser cavity was truncated by the lens aperture to produce a well-defined collimated laser beam. Further, the radius of the returned beam at the laser front facet was much larger than the size of the active layer due to 


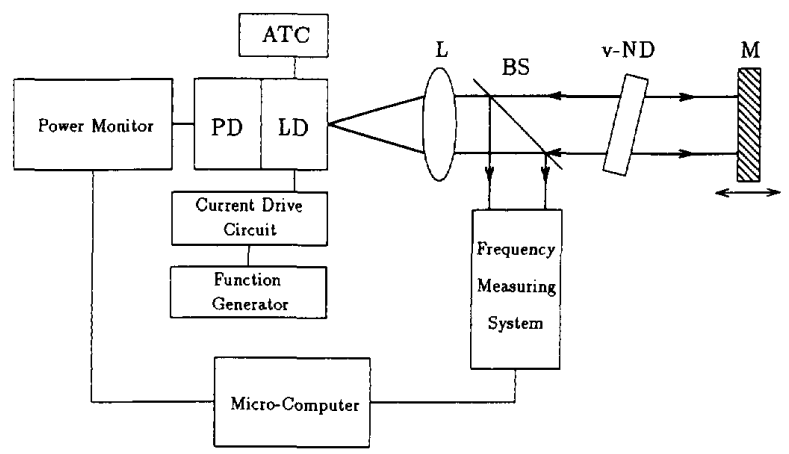

Fig. 5. Experimental setup for measuring the characteristics of a semiconductor laser with external optical feedback.

the diffraction. Therefore, the actual feedback intensity to the laser cavity was reduced to one over several tens or roughly one-hundredth of our employed feedback ratio. It is shown later that our experimental data can bè compared with the theoretical results for the feedback-intensity ratio of about one-hundredth of the experimental ratio.

Fig. 6 shows the observed output power for the injection current change. The bias current of the laser was 70 $\mathrm{mA}$ at $20^{\circ} \mathrm{C}$ and the injection current of the laser was modulated by a triangular wave at a frequency of $200 \mathrm{~Hz}$. In the following discussion, the same conditions for the bias injection current, the temperature, and the modulation frequency were used. The modulation width of the injection current was $\pm 1 \mathrm{~mA}$. It was confirmed that there were no mode hops originating from the internal-mode transitions within the modulation-current range at that temperature. The undulation of the output power with the increase of the injection current can be seen in the figure. At the same external-cavity length, the undulation can be clearly observed with an increase of the feedback intensity, as shown in Fig. 6 (b) and (c). From Fig. 6 (b), one can see the hysteresis in the output power. The same results have been observed in previous work [5]. But the effect is reduced for a long-external-cavity length at the same intensity reflectance. In Fig. 6 (c), the amplitude of the undulation of the output power becomes small compared with that of Fig. 6 (b). The period of undulation is roughly half that in Fig. 6 (a). This effect is explained from the oscillations of several submodes, as shown later. Fig. 7 shows the calculated results of the output power against the injection current for different external-cavity lengths. The amplitude of the undulation becomes larger for a larger intensity reflectance. The period of undulation becomes small with an increase of the external-cavity length. This tendency qualitatively coincides with the observed results in Fig. 6. Remarkable bistable states are seen in the output power, especially for the case of a large-feedback ratio. But the amplitudes of the undulation remain unchanged as long as the intensity reflectances are the same, under the assumption of singlemode oscillation of the laser. This result is discrepant with

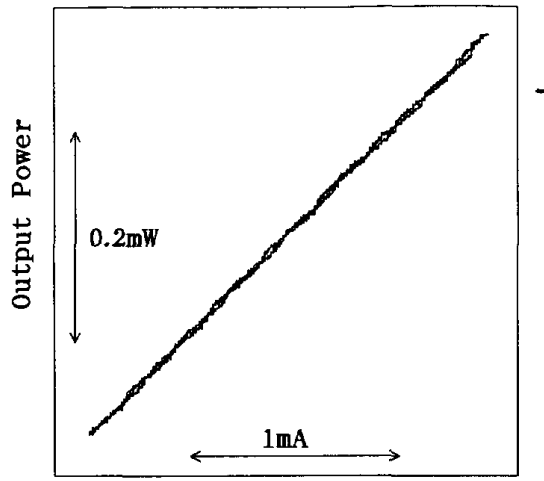

Injection Current

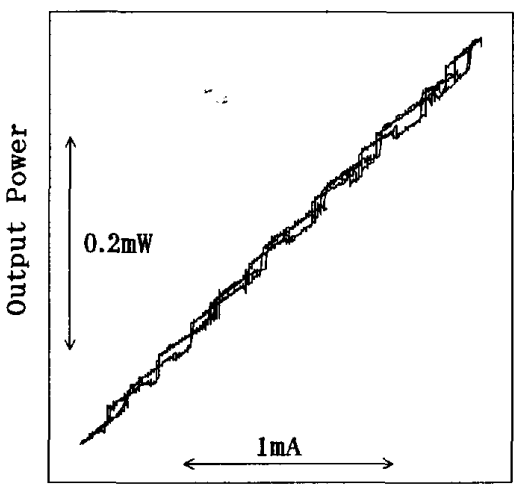

Injection Current

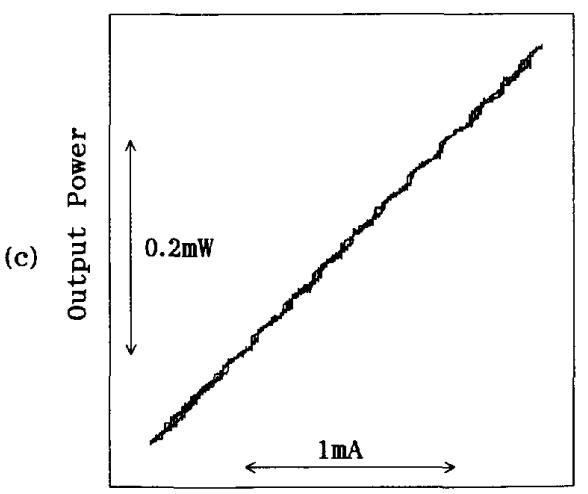

Injection current

Fig. 6. Observed-output power for the injection current. (a) $R_{3}=0.0905$ and $L=5 \mathrm{~cm}$; (b) $R_{3}=0.387$ and $L=5 \mathrm{~cm}$; and (c) $R_{3}=0.387$ and $L=10 \mathrm{~cm}$.

the observed experimental data in Fig. 6. As already discussed, the values of the intensity-reflectance ratio used in the theoretical calculations are as small as onehundredth of those for the experiments.

The dependencies of the laser-oscillation frequency on the intensity reflectance and the external-cavity length were studied. Fig. 8 shows the laser-oscillation frequencies monitored through the Fabry-Perot interferometer plotted as functions of the intensity reflectance and the injec- 
(a)

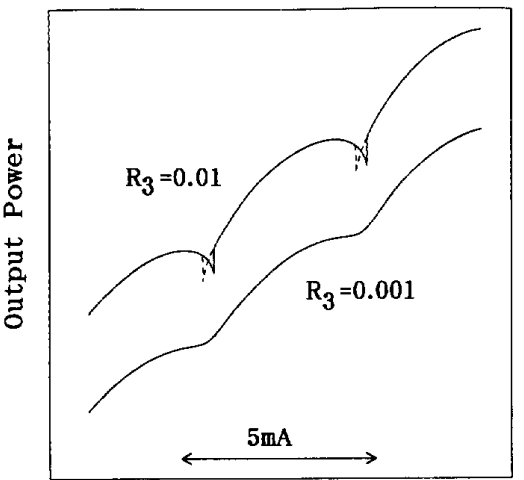

Injection Current

(b)

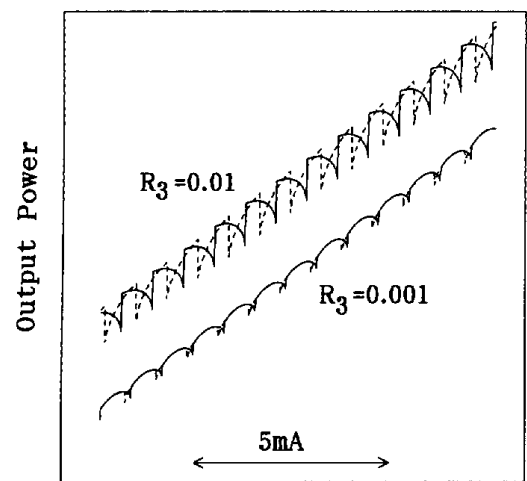

Injection current

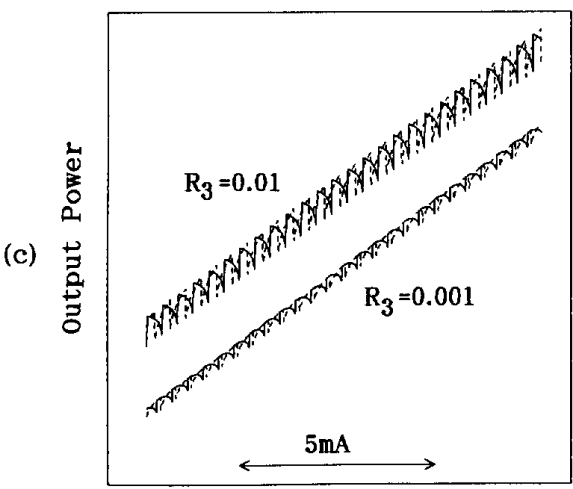

Injection Current

Fig. 7. Calculated results of the output power versus the injection current for different external-cavity lengths. (a) $L=10 \eta l$, (b) $L=50$ $\eta l$, and (c) $L=100 \eta l$.

tion current. The free-spectral range of the Fabry-Perot interferometer used was $7.5 \mathrm{GHz}$. Without external feedback, the laser frequency changes linearly with a change in the injection current, and the frequency modulation efficiency $I_{r}$ defined in (20) was measured to be 3.85 $\mathrm{GHz} / \mathrm{mA}$. The horizontal axis shown in the figure represents the frequency. At a short-external-cavity length, the mode of the oscillation becomes rather instable and the mode hop proportional to the external-cavity length

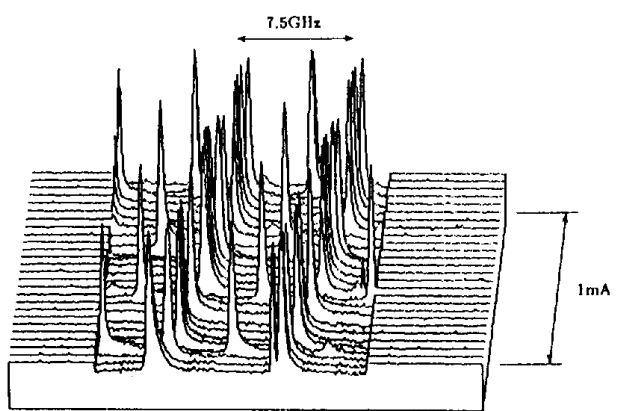

(a)

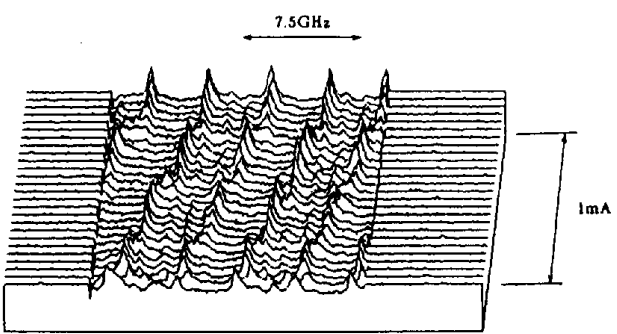

(b)

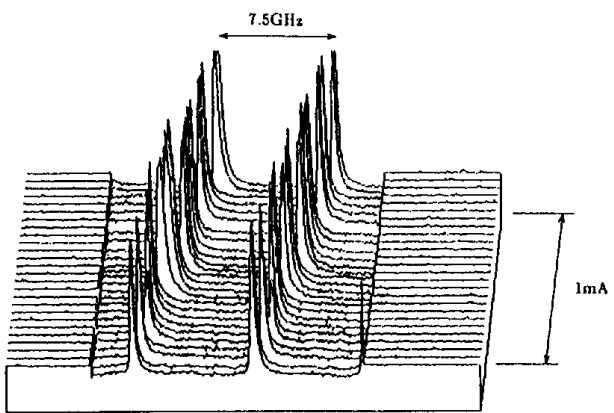

(c)

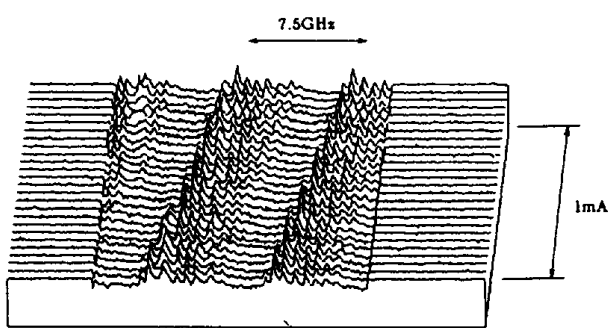

(d)

Fig. 8. Oscillation frequency observed by the Fabry-Perot interferometer for the injection-current change. The horizontal axis represents the frequency. (a) $R_{3}=0.0297$ and $L=10 \mathrm{~cm}$; (b) $R_{3}=0.387$ and $L=10$ $\mathrm{cm}$; (c) $R_{3}=0.0297$ and $L=20 \mathrm{~cm}$; and (d) $R_{3}=0.387$ and $L=20$ $\mathrm{cm}$.

(around $1 \mathrm{GHz}$ for a $10 \mathrm{~cm}$ external-cavity length) can be seen in Fig. 8 (a). With an increase in the external-feedback optical power, the laser oscillates as if it were a multimode oscillation with an external-cavity spacing as shown in Fig. 8 (b). But, for the longer external-cavity length shown in Fig. 8 (c), the oscillation becomes stable 
compared with that in Fig. 8 (a), even for the same intensity reflectance. With a larger feedback power and a longer external-cavity length, the laser oscillation appears stable and has many submodes whose spacings are equal to the external-cavity length shown in Fig. 8 (d).

It is not easy to conduct a quantitative study of laser oscillation for the results obtained in the Fabry-Perot experiments. Therefore, the frequency change of laser oscillation due to external optical feedback was investigated by using a Twyman-Green interferometer in the experimental setup shown in Fig. 5. The injection current to the laser was modulated by a triangular signal. Then the interference output $P_{\mathrm{TG}}$ from the Twyman-Green interferometer is given by [11]

$$
P_{\mathrm{TG}}(I)=P(I)\left\{1+V \cos \left[\frac{2 D}{c} \Omega(I)\right]\right\}
$$

where $P(I)$ is the power proportional to the laser power in (14), $V$ is the fringe visibility of the interferometer output, and $D$ is the difference of the lengths between the interferometer arms. Here, the power $P$ and the frequency $\Omega$ are described as explicit functions of the injection current. From this relation, we can investigate the change of the oscillation frequency more precisely. Without external feedback, the interference output power is as shown in Fig. 9 (a). The upper part of the figure shows the time signal of the triangular injection current modulation and the lower part shows the result of the output power. The output power varies with an increase of injection current as is easily understood from (28), because the laser-output power is linearly proportional to the injection current. The amplitude of the modulation is $\pm 1 \mathrm{~mA}$ and its frequency is $200 \mathrm{~Hz}$. The peak-to-valley value of the modulation signal corresponds to $7.70 \mathrm{GHz}$ for the laser-oscillation frequency. The length $D$ of the difference of the interferometer arms was chosen to be about $5 \mathrm{~mm}$. Fig. 9 (b) and (c) show the results with external feedback at the same feedback power. With an increase of external-cavity length, the width of the small periodic undulation in the modulated power increases. This small undulation corresponds to a change of the oscillation mode when the external-feedback light is returned to the laser cavity. The mode hop of the oscillation frequency can be calculated from the number $p$ of the undulations included within one-half of the frequency of the triangular signal as

$$
\delta \nu=\frac{I_{r} \delta I}{p}
$$

where $\delta I$ is the modulation depth of the current. The expected mode hop $\delta \nu$ is calculated from (21). The frequency of the mode hop is experimentally obtained as $0.96 \mathrm{GHz}$ at an external-cavity length $L=15 \mathrm{~cm}$, whereas the expected mode hop is $1.00 \mathrm{GHz}$. This is a positive coincidence. It is not clear, but the hysteresis of the periodic waves in the power can be seen by closely looking at the figure.
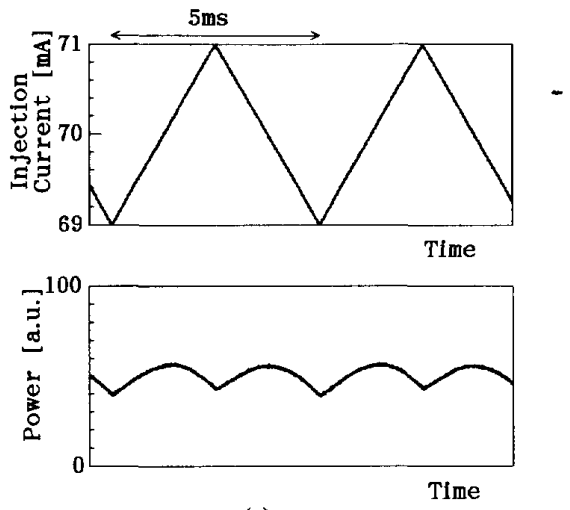

(a)
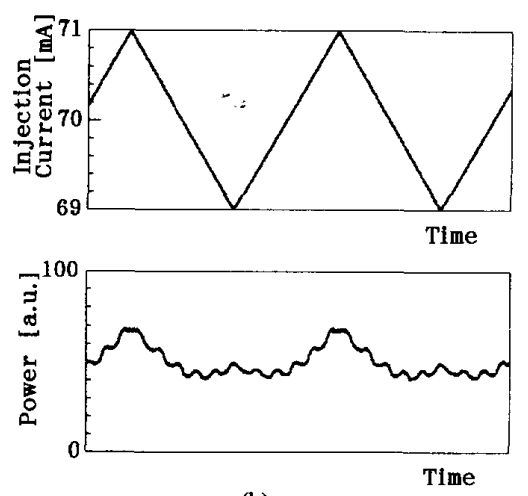

(b)
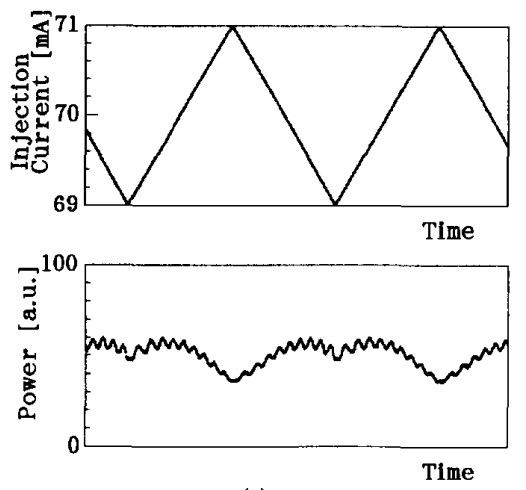

(c)

Fig. 9. Interference output from the Twyman-Green interferometer. (a) $R_{3}=0$; (b) $R_{3}=0.00620$ and $L=7.5 \mathrm{~cm}$; and (c) $R_{3}=0.00620$ and $L=15 \mathrm{~cm}$.

The theoretical calculation of the interferometer output power for the modulation of the driving current was achieved by substituting (21) and (28) and is shown in Fig. 10 . The values of the parameters used in the calculations were almost compatible with those of the experiments. Fig. 10 (a) shows the modulation signal of the triangular wave and Fig. 10 (b) shows the interference output power from the Twyman-Green interferometer without external feedback. Fig. 10 (c) and (d) show the interference output power for different external-cavity lengths but for the 


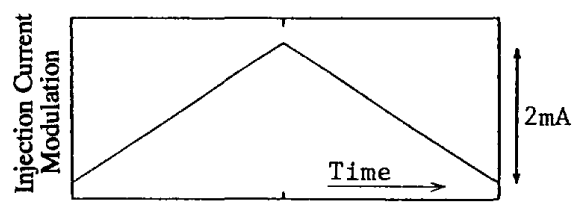

(a)

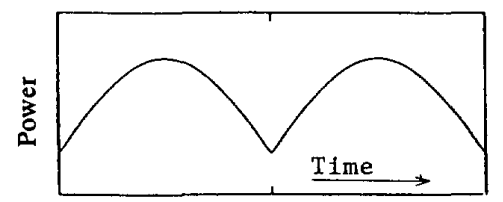

(b)

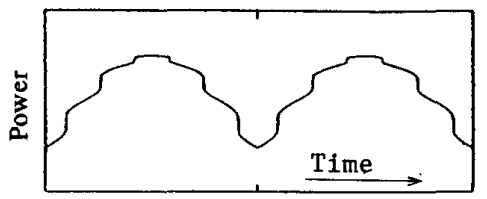

(c)

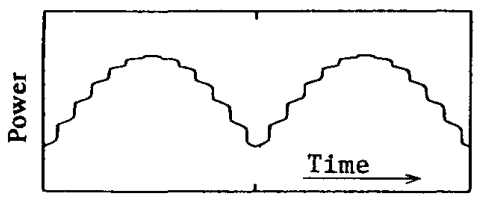

(d)

Fig. 10. Theoretical calculation of the interference output from the Twyman-Green interferometer. (a) Injection current modulation. (b) Output power at $R_{3}=0$. (b) $R_{3}=0.0001$ and $L=100 \eta l$. (c) $R_{3}=$ 0.0001 and $L=200 \mathrm{\eta l}$.

same intensity reflectance. By considering the previously mentioned differences in the values of the external-mirror reflectances between the experiments and the theoretical calculations, the qualitative agreement between the experimental and theoretical results is seen.

The dependence of the laser-output power on the change of the external-cavity length was studied. At the range from 15 to $19 \mathrm{~mm}$ from the front facet of the laser cavity, the external-cavity length was changed by moving the external mirror on the motor-driven table. The results are shown in Fig. 11 for different external-feedback intensities. At small optical feedback, as shown in Fig. 11 (a), the dips of the laser-output power appear periodically, as discussed in Section II-D. The period of undulation in the output power is about $1.2 \mathrm{~mm}$. Using the values of the cavity length $\sim 300 \mu \mathrm{m}$ of the laser diode and the calculated effective refractive index $\eta_{\text {eff }} \sim 4$, the effective-laser length is roughly estimated as $1.2 \mathrm{~mm}$. The estimated length agrees well with the experimental period. However, the effective-refractive index is strongly dependent on the respective materials and the device, so that this device parameter must be directly measured for a more precise comparison. In Fig. 11 (a), it is considered that two modes are mainly oscillating as assumed in the previous section. At the same external-cavity length but

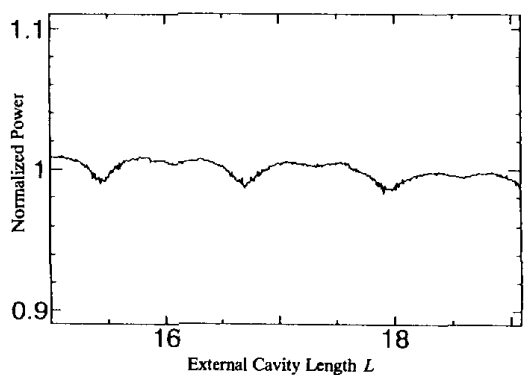

(a)

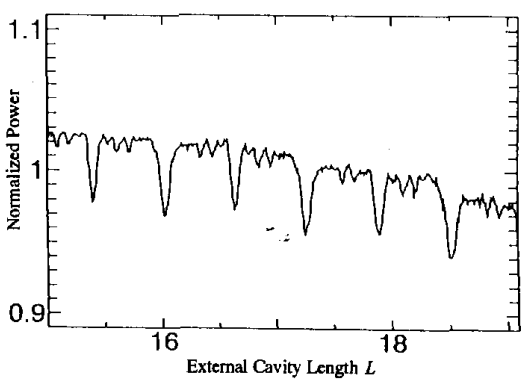

(b)

Fig. 11. Experimental results of the output power as a function of the external-cavity length for different intensity reflectances. (a) $R_{3}=0.0115$ and $L=15 \sim 19 \mathrm{~mm}$ and (b) $R_{3}=0.387$ and $L=15 \sim 19 \mathrm{~mm}$.

with larger optical feedback, many large dips are observed within the effective-laser length due to multimode laser oscillation, as shown in Fig. 11 (b).

Fig. 12 also shows the dependence of the output power on the variations of the external-cavity length. The difference between Fig. 12 (a) and (b) is the location of the external mirror. The external mirror is located a small distance from the laser cavity in Fig. 12 (a), while it is located a rather long distance away in Fig. 12 (b). The ranges of the external-cavity variations for both figures are the same. When the external cavity is not located as far from the laser cavity, we can observe dips due to mode interference. However, only a slight undulation can be seen in the laser output power when the external cavity is located far from the laser cavity. One of the possible reasons is the degradation of the coherence of the laser oscillation. But the coherence length, in the usual sense, is much longer than the length treated in Fig. 12 (b). We consider another reason for the disappearance of the dips at a long-external-cavity length as follows. Here we assume that the laser oscillates at only two internal modes, but with each mode containing accompanying external modes. Fig. 13 shows the schematic illustration of the phase condition calculated from (21) and the internal-laser modes for a certain external feedback. The horizontal axis is the frequency. From (21), the phase condition of laser oscillation with the external cavity is satisfied at the intersections of the periodic function and the linear curve [6], as shown in the figure. If the periods of the internal and external modes coincide with each other, and the external 


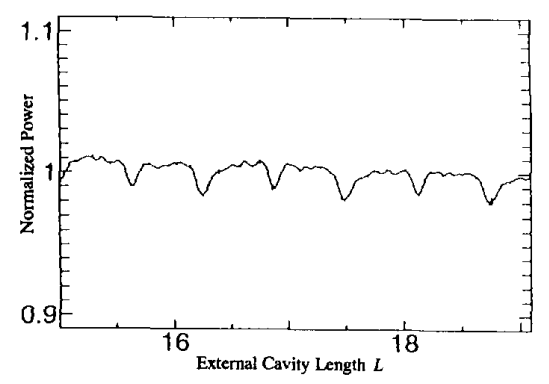

(a)

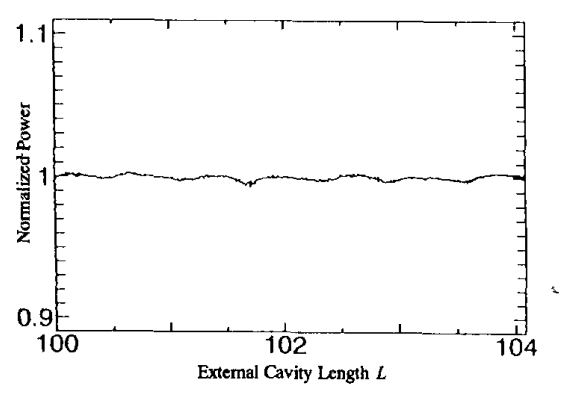

(b)

Fig. 12. Experimental results of the output power as a function of the external-cavity length for different cavity locations. (a) $R_{3}=0.248$ and $L=15 \sim 19 \mathrm{~mm}$ and (b) $R_{3}=0.248$ and $L=100 \sim 104 \mathrm{~mm}$.

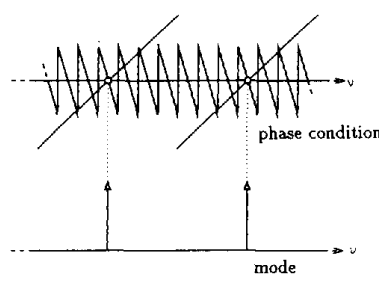

(a)

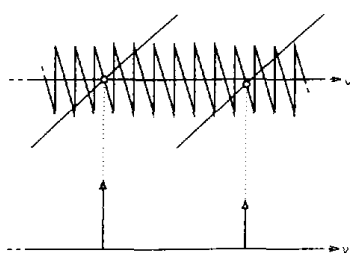

(b)
Fig. 13. Schematic illustrations of the phase condition (upper) and the internal-mode condition (lower) for short feedback length or rather-small optical feedback. (a) Mode agreement condition. (b) Mode disagreement condition.

modes oscillate at integer multiples of the internal mode as shown in Fig. 13 (a), each internal mode oscillates at its maximum power and the total laser output power is maximized. On the other hand, for example, if one or neither of the internal modes satisfies the maximum gain condition as shown in Fig. 13 (b), the total output power decreases. For large external optical feedback, there is a great possibility of multimode laser oscillation. This situation corresponds to Fig. 11 (a). On the other hand, when the external-cavity length is large, the powers of the adjacent modes become small and the laser oscillates at multimode. The situation corresponds to Fig. 12 (b) and is illustrated in Fig. 14. In this case, many submodes accompanying the internal modes oscillate due to the external feedback. Fig. 14 (a) shows the case where the externalcavity length coincides with integer multiples of the internal-cavity length. Fig. 14 (b) shows the case for a discrep-

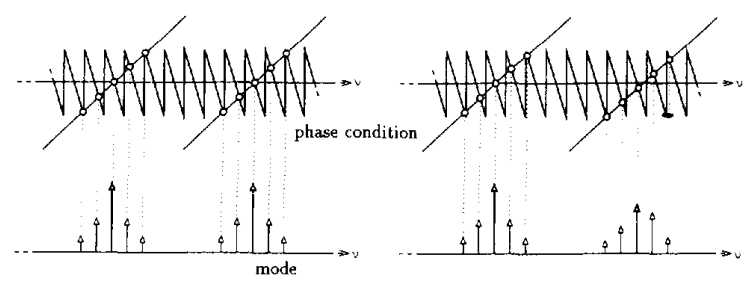

(a)

(b)

Fig. 14. Schematic illustrations of the phase condition (upper) and the internal-mode condition (lower) for long feedback length or large optical feedback. (a) Mode agreement condition. (b) Mode disagreement condition.

ancy between the internal and external mode lengths. In the cases shown in Fig. 14, the differences of the total output powers are reduced due to the presence of many oscillation modes. The consideration here gives a qualitative explanation for the experimental results in this paper.

\section{CONCLUSIONS}

We have discussed the characteristics of a semiconductor laser with external optical feedback by using a compound-cavity model. The oscillation frequency and gain conditions of the compound cavity have been derived by considering multiple reflections of light within the external cavity. The oscillation characteristics are strongly dependent on the feedback intensity and the external-cavity length. The theoretical explanations for the experimentally observed results have been given and a qualitative agreement between the theory and the experiment has been obtained. Thus, it is proved that the characteristics of the oscillation of a semiconductor laser in the presence of external optical feedback can be explained by the rate equations by considering multiple reflections of light in the external cavity, even for large optical feedback.

We have assumed two modes oscillation for small and long external feedback lengths to explain the experimental results. However, we have neglected mode competition or multimode oscillation of more than two modes. In actuality, such is the possible case for large optical feedback. Therefore, multimode oscillation and the mode competition should be taken into account for precise comparisons and further considerations of an exact theory for the compound cavity. We also neglected the noise induced by optical feedback which, in actuality, is related to mode competition. The noise induced by optical feedback plays an important role in laser oscillation of the compound cavity.

\section{ACKNOWLEDGMENT}

The authors wish to thank Y. Liu for helpful discussions.

\section{REFERENCES}

[1] G. A. Acket, D. Lenstra, A. J. den Boef, and B. Verbeek, "The influence of feedback intensity on longitudinal mode properties and optical noise in index-guided semiconductor lasers," IEEE $J$. Quantum Electron., vol. QE-20, pp. 1163-1169, 1984. 
[2] T. Aoshima and J. Ohtsubo, "Two-dimensional vector LDV using laser diode frequency change and self-mixing effect," Opt. Commun., vol. 92, pp. 219-224, 1992 .

[3] M. H. Koelink, M. Slot, F. F. M. de Mul, J. Greve, R. Graaff, A. C M. Dassel, and J. G. Aarnoudse, "Laser Doppler velocimeter based on the self-mixing effect in a fiber-coupled semiconductor laser: Theory," Appl. Opt., vol. 31, pp. 3401-3408, 1992

[4] A. P. Bogatov, P. G. Eliseev, L. P. Ivanov, A. S. Logginov, J. A Manko, and K. Ya. Senatorov, "Study of the single-mode injection laser," IEEE J. Quantum Electron., vol. QE-9, pp. 392-398, 1973.

[5] R. Lang and K. Kobayashi, "External optical feedback effects on semiconductor injection laser properties," IEEE J. Quantum Electron., vol. QE-16, pp. 347-355, 1980.

[6] J. H. Osmundsen and N. Gade, "Influence of optical feedback on laser frequency spectrum and threshold conditions," IEEE J. Quantum Electron., vol. QE-19, pp. 465-469, 1983.

[7] T. Fujita, S. Ishizuka, K. Fujito, H. Serizawa, and H. Sato, "Intensity noise suppression and modulation characteristics of a laser diode coupled to an external cavity," IEEE J. Quantum Electron., vol. QE-20, pp. 429-499, 1984.

[8] H. Sato, T. Fujita, and K. Fujito, "Intensity fluctuation in semiconductor lasers coupled to external cavity," IEEE J. Quantum Electron., vol. QE-21, pp. 46-51, 1985.

[9] G. C. Dent, P. S. Durkin, K. A. Wilson, and C. E. Moeller, "Chaos in the coherence collapse of semiconductor lasers," IEEE J. Quantum Electron., vol. 24, pp. 2441-2447, 1988.

[10] M. Yamada, "Transverse and longitudinal mode control in semiconductor injection lasers," IEEE J. Quantum Electron., vol. QE-19, pp. 1365-1380, 1983.

[11] J. Ohtsubo and Y. Liu, "Optical bistability and multistability in an active interferometer," Opt. Lett., vol. 15, pp. 731-733, 1990.

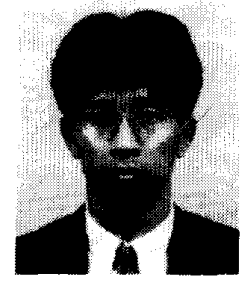

Hiroshi Kakiuchida was born in Ishikawa, Japan, in 1968 . He received the B.S. and M.S. degrees in opto-electronics and mechanical engineering, both from Shizuoka University, Shizuoka,Japan, in 1991 and 1993, respectively.

He is now with Dainippon Screen Co. Ltd. Kyoto, Japan.

Mr. Kakiuchida is a member of the Japanese Society of Applied Physics.

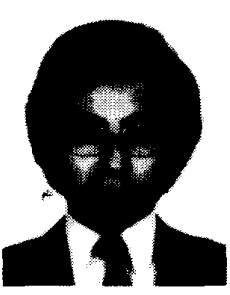

Junji Ohtsubo received the B.S. degree in electronics from the Kyusyu Institute of Technology in 1973 and the M.S. and Ph.D. degrees in electronics from Hokkaido University in 1975 and 1978 , respectively.

In 1978, he joined the Mechanical Engineering Laboratory. During 1981-1982, he was a Research Associate at the Institute of Optics University of Rochester. He joined Shizuoka

University, Shizuoka, Japan, as an Associate Professor in 1985 and is presently a Professor. His current research interests are statistical optics, speckle, optical inspections, active interferometry, optical information processing and computing, and nonlinear dynamics in optics.

Prof. Ohtsubo is a member of the Optical Society of America, SPIE, the Japanese Society of Applied Physics, and the Laser Society of Japan. 